Preprint

UCRL-JC-149639

\title{
Improved Optical Design for the Large Synoptic Survey Telescope (LSST)
}

\section{L.G. Seppala}

This article was submitted to Society of Photo-Optical Instrumentation Engineers Conference on Astronomical Telescopes and Instrumentation, Waikoloa, HI, August 22-28, 2002

\section{September 24, 2002}

U.S. Department of Energy

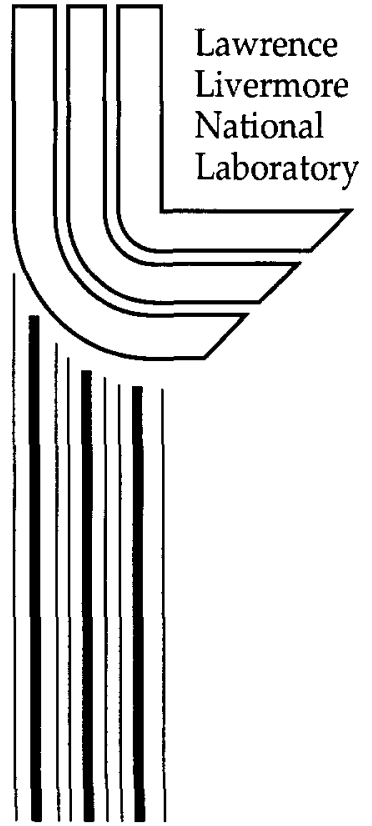




\section{DISCLAIMER}

This document was prepared as an account of work sponsored by an agency of the United States Government. Neither the United States Government nor the University of California nor any of their employees, makes any warranty, express or implied, or assumes any legal liability or responsibility for the accuracy, completeness, or usefulness of any information, apparatus, product, or process disclosed, or represents that its use would not infringe privately owned rights. Reference herein to any specific commercial product, process, or service by trade name, trademark, manufacturer, or otherwise, does not necessarily constitute or imply its endorsement, recommendation, or favoring by the United States Government or the University of California. The views and opinions of authors expressed herein do not necessarily state or reflect those of the United States Government or the University of California, and shall not be used for advertising or product endorsement purposes.

This is a preprint of a paper intended for publication in a journal or proceedings. Since changes may be made before publication, this preprint is made available with the understanding that it will not be cited or reproduced without the permission of the author.

This report has been reproduced directly from the best available copy.

Available electronically at http://www.doc.gov/bridge

Available for a processing fee to U.S. Department of Energy

And its contractors in paper from

U.S. Department of Energy

Office of Scientific and Technical Information

$$
\text { P.O. Box } 62
$$

Oak Ridge, TN 37831-0062

Telephone: (865) 576-8401

Facsimile: (865) 576-5728

E-mail: reports@adonis.osti.gov

Available for the sale to the public from

U.S. Department of Commerce

National Technical Information Service 5285 Port Royal Road Springfield, VA 22161

Telephone: (800) 553-6847

Facsimile: (703) 605-6900

E-mail: orders@ntis.fedworld.gov

Online ordering: http://www.ntis.gov/ordering.htm

OR

Lawrence Livermore National Laboratory

Technical Information Department's Digital Library

http://www.llnl.gov/tid/Library.html 


\title{
Improved optical design for the Large Synoptic Survey Telescope (LSST)
}

\author{
Lynn G. Seppala, University of California, Lawrence Livermore National Laboratory
}

\begin{abstract}
This paper presents an improved optical design for the LSST, an $\mathrm{f} / 1.25$ three-mirror telescope covering 3.0 degrees full field angle, with $6.9 \mathrm{~m}$ effective aperture diameter. The telescope operates at five wavelength bands spanning $386.5 \mathrm{~nm}$ to $1040 \mathrm{~nm}$ (B, V, R, I and Z). For all bands, $80 \%$ of the polychromatic diffracted energy is collected within 0.20 arcseconds diameter. The reflective telescope uses an $8.4 \mathrm{~m} \mathrm{f} / 1.06$ concave primary, a $3.4 \mathrm{~m}$ convex secondary and a $5.2 \mathrm{~m}$ concave tertiary in a Paul geometry. The system length is $9.2 \mathrm{~m}$. A refractive corrector near the detector uses three fused silica lenses, rather than the two lenses of previous designs. Earlier designs required that one element be a vacuum barrier, but now the detector sits in an inert gas at ambient pressure. The last lens is the gas barrier. Small adjustments lead to optimal correction at each band. The filters have different axial thicknesses. The primary and tertiary mirrors are repositioned for each wavelength band. The new optical design incorporates features to simplify manufacturing. They include a flat detector, a far less aspheric convex secondary ( $10 \mu \mathrm{m}$ from best fit sphere) and reduced aspheric departures on the lenses and tertiary mirror. Five aspheric surfaces, on all three mirrors and on two lenses, are used. The primary is nearly parabolic. The telescope is fully baffled so that no specularly reflected light from any field angle, inside or outside of the full field angle of 3.0 degrees, can reach the detector.
\end{abstract}

Keywords: astronomical telescopes, wide field telescopes, three mirror telescopes, survey telescopes, optical design, telescope design, telescope baffles

\section{SPECIFICATIONS}

The Large-aperture Synoptic Survey Telescope (LSST) is a proposed 8.4 meter diameter, 7 degree $^{2}$ field telescope. The product of collecting area and field of view will be 20 times greater than any observatory now operating or under construction. An array of CCD totaling several giga-pixels will collect data with expected exposure times of 10 to 20 seconds. This telescope will survey large portions of the sky to visual magnitudes of 24 or more, searching for Earththreatening asteroids or probing the nature of dark energy. Good telescopes at the best sites will deliver images of 0.5 arc-sec on occasion, more frequently in the near infrared. The LSST should perform significantly better than this for each photometric spectral band to avoid further degradation of the image. The pixel sampling should be at least 0.25 arc$\sec$ (the Nyquist sampling criterion). Using this criterion, a 1.4 giga-pixel array is needed. About 1.0 terabyte of data could be collected per viewing night.

Table 1 summarizes the parameters of recent designs. The LSST uses an $8.4 \mathrm{~m}$ diameter primary in a telescope with a $10.5 \mathrm{~m}$ focal length. The focal ratio is $\mathrm{f} / 1.25$. A detector $55 \mathrm{~cm}$ in diameter is necessary for a full 3 degrees field of view. The area obscuration on-axis is $25 \%$, rising to $38 \%$ at full field. The telescopes were designed to operate at five photometric spectral bands. Spectral filters are thus required. Since the filter and any required window lead to chromatic focal shifts and aberrations, aspheric lens correctors were used to compensate these effects. With these modifications, one-third arc-second images could be achieved over all bands.

\begin{tabular}{|l|l|l|l|}
\hline PARAMETER & VALUE & PARAMETER & VALUE \\
\hline Maximum diameter & 8.4 meters & Maximum length & 9.0 meters \\
\hline Focal length & 10.5 meters & Focal ratio & $\mathrm{f} / 1.25$ \\
\hline Plate scale & $51 \mu \mathrm{m} /$ arc-sec & Focal surface & weakly curved aspheric \\
\hline Full field of view & 3.0 degrees & Detector diameter & 0.55 meters \\
\hline Design type & 3 -mirror Paul-Baker & Design modifications & two corrector lenses \\
\hline Obscuration & $25 \%$ on axis & Obscuration & $38 \%$ full field \\
\hline
\end{tabular}

Table 1. First order optical specifications for previous versions of the LSST telescope. 
Additional specifications and proposed operating conditions along with science goals are summarized in this conference proceedings. $^{1}$

\section{PREVIOUS DESIGN HISTORY}

The history of three-mirror telescopes that cover wide fields goes back to $\mathrm{M}$. Paul ${ }^{2}$ in 1935 , as nicely summarized by Wilson $^{3}$. Paul recognized that a Mersenne telescope, a pair of concave and convex paraboloids, was corrected for spherical aberration, coma and astigmatism. Knowledge of basic aberration theory led to a three-mirror design, corrected for these three aberrations, in which both the secondary and tertiary mirrors are spherical. A spherical tertiary mirror of equal but opposite curvature of the secondary mirror was added to form a real focus. The tertiary mirror was placed with its center of curvature at the vertex of the secondary mirror. The spherical aberration correction required for the tertiary mirror was achieved by removing the parabolic figure of the secondary. In effect, this three-mirror reflector is equivalent to a refractive Schmidt-Cassegrain telescope. There are few degrees of freedom, given that the beam is collimated between the secondary and tertiary. Once the primary mirror diameter and focal ratio are chosen, the only remaining degree of freedom is the beam reduction ratio on the secondary. Paul chose this reduction ratio to ensure that the tertiary mirror was located in front of the primary. Baker ${ }^{4}$ and Willstrop ${ }^{5}$ refined the Paul design to create telescopes with larger fields of view. The tertiary was placed well behind the primary mirror. Baker allowed the secondary mirror to have an aspheric profile and removed the restriction of matching the curvature of the tertiary. As a result, a flat field could be achieved. Telescopes of a few meters in diameter, operating around $f / 2$ to $f / 3$ and covering 3 or 4 degree fields, could achieve near diffraction-limited performance. Angel, Lessor and Sarlot ${ }^{6}$ demonstrated that a compact telescope design operating at a faster focal ratio and covering a wide field could satisfy the demanding imaging performance required by LSST. Angel et al added two corrector lenses to compensate for chromatic shifts of the spectral filter to achieve one-third arc-second images. All these designs have collimated beams between the secondary and tertiary. The field angle is set by a combination of imaging performance, baffling against stray light and the increased obscuration due to baffles off-axis.

\section{CURRENT DESIGN STUDY PARAMETERS}

A design study was undertaken starting about 15 months ago to determine if improvements to the existing LSST design could be achieved. The first-order design was generally maintained, since it met the scientific goals and produced a relatively compact design. However, the list of parameters explored gradually grew. The number, complexity in terms of polynomial terms and distribution of aspheric surfaces were examined. The focal ratio of the primary was allowed to vary. The restriction of a collimated beam reflected from the secondary was lifted, which led to changes in the on-axis obscuration and required baffles. All designs were required to be fully baffled such that no directly-reflected light from the tertiary could reach the detector. Previous designs were baffled so that no light from within the specified field of view could reach the detector. Some light, although badly aberrated, could reach the detector from field angles exceeding the full field angle. Finally, an extra lens element was added near the detector to form a triplet lens corrector.

Several strategies helped to maintain a solution that is reasonable to fabricate and test. Aspheric surfaces were simplified in a number of ways. A minimum number of aspheric surfaces was used. In addition, high order aspheric terms were used only whenever necessary. As aspheric terms are added, more rays and field points are required during optimization to ensure a valid figure of merit for the optimization procedure. If too many terms were allowed, steep aspheric gradients could result. Aspheric departures from best-fit spheres were monitored and controlled during optimization. In particular, close attention was paid to minimizing the aspheric departure on the convex secondary to ease fabrication and testing. Finally, reasonable center and edge thicknesses were maintained. Edge thicknesses of positive lenses and the center thickness of the negative lens were maintained at $2 \mathrm{~cm}$. The plane parallel spectral filters were required to be at least 1.25 $\mathrm{cm}$ thick.

Table 2 shows parameters that significantly changed during the design evolution. The focal ratio of the primary increased slightly, from $\mathrm{f} / 1.0$ to $\mathrm{f} / 1.07$. In addition, the beam became divergent at $\mathrm{f} / 12$ after reflection from the secondary mirror. The expanding beam from the secondary required several associated changes to maintain $38 \%$ obscuration at full field. The hole in the primary mirror had to get larger to avoid excessive vignetting. As a result, the on-axis area obscuration for this design increased from $25 \%$ to $30 \%$ and the tertiary mirror grew in diameter by 0.3 meters. An extra lens was added to form a triplet lens corrector. The secondary asphericity has been substantially reduced to only 10 microns from the best-fit sphere. The focal surface tended to be almost flat, so that a flat focal plane could be specified without penalizing optical imaging. The five spectral filters were allowed to have different thicknesses. 


\begin{tabular}{|l|l|l|}
\hline Parameter & Earlier designs & New design \\
\hline Primary focal ratio & $\mathrm{f} / 1.0$ & $\mathrm{f} / 1.07$ \\
\hline Beam divergence between secondary and tertiary & collimated & $\mathrm{f} / 12$ diverging \\
\hline \multicolumn{1}{|c|}{ tertiary diameter } & $4.9 \mathrm{~m}$ & $5.2 \mathrm{~m}$ \\
\hline area obscuration: on-axis & $25 \%$ & $30 \%$ \\
\hline area obscuration: full field & $38 \%$ & $38.6 \%$ \\
\hline Number of corrector lenses & 2 & 3 \\
\hline Secondary mirror: aspheric departure from best-fit sphere & $>150$ microns & $<10$ microns \\
\hline Focal plane surface & curved aspheric & flat \\
\hline Thickness of spectral filters & constant & varies: $12.5 \mathrm{~mm}$ to $21 \mathrm{~mm}$ \\
\hline Images: $80 \%$ polychromatic diffraction & $<0.33$ arc-sec & $<0.21$ arc-sec \\
\hline
\end{tabular}

Table 2. Significant changes in the new telescope design

There are several reasons for the improvement in performance of this telescope. Increasing the focal ratio of the primary, from $\mathrm{f} / 1.0$ to $\mathrm{f} / 1.07$, reduces aberrations that must be compensated elsewhere in the telescope. Allowing the beam to diverge at $\mathrm{f} / 12$ from the secondary has similar advantages. The effective focal ratio of the secondary goes from $\mathrm{f} / 1.0$ to $\mathrm{f} / 1.09$, and of the tertiary from $\mathrm{f} / 1.25$ to $\mathrm{f} / 1.4$. Table 3 shows the amount of reduction in primary wavefront aberrations for each of the mirrors, assuming that the contributions scale as a power of the effective focal ratios. One sees that third order aberrations are reduced by $20 \%$ to $36 \%$.

\begin{tabular}{|l|l|l|l|l|l|}
\hline Mirror & $\begin{array}{l}\text { Old effective } \\
\text { focal ratio }\end{array}$ & $\begin{array}{l}\text { New effective } \\
\text { focal ratio }\end{array}$ & $\begin{array}{l}\text { Reduction in } \\
4^{\text {th }} \text { order (SA3) }\end{array}$ & $\begin{array}{l}\text { Reduction in } \\
3^{\text {rd }} \text { order(CMA3) }\end{array}$ & $\begin{array}{l}\text { Reduction in } \\
2^{\text {nd }} \text { order(AST3) }\end{array}$ \\
\hline Primary & 1.0 & 1.07 & $24 \%$ & $18 \%$ & $13 \%$ \\
\hline Secondary & 1.0 & 1.09 & $29 \%$ & $23 \%$ & $16 \%$ \\
\hline Secondary & 1.25 & 1.40 & $36 \%$ & $29 \%$ & $20 \%$ \\
\hline
\end{tabular}

Table 3. Estimates of aberration reduction based on increasing the effective focal ratios of each mirror

A triplet lens corrector has sufficient design variables to correct axial and lateral chromatic aberrations and to control all monochromatic third-order aberrations. Significant improvements in image sizes were realized with the addition of a third lens corrector. An added bonus was that the focal surface was almost flat. No penalty was incurred for a flat focal surface. Finally, each plano spectral filter was allowed to have a different thickness. In particular, because of dispersion, the aberrations and focal lengths of the lenses change with wavelength. Slight aberration differences could be introduced by changing the filter thickness and helped specifically to achieve uniform performance from band to band.

\section{IMPROVED OPTICAL PERFORMANCE AND MANUFACTURABILITY}

Figure 1 shows the imaging performance of the new design for each of the photometric spectral bands $B, V, R, I$ and $Z$. The diameter of a focal spot that includes $80 \%$ of the polychromatic diffracted energy, as a function of radial position across the detector, is plotted. Five wavelengths, equally spanning each spectral band, were included in this analysis. For comparison, the $80 \%$ collection for a diffraction-limited telescope in the infra-red $Z$ band is also plotted $(0.083$ arcseconds). The Z-band has images approximately twice the diffraction limit. Overall, the energy collection is within 0.20 arc-seconds. The energy collection of this design is quite uniform across the field for each spectral band and does not vary significantly between spectral bands. The extreme bands show the worst performance. In the near-ultraviolet $B$ band, the lenses are very dispersive, limiting performance for a $100 \mathrm{~nm}$ band. In the infra-red $\mathrm{Z}$ band, a bandwidth of 200 $\mathrm{nm}$ has comparable performance. 


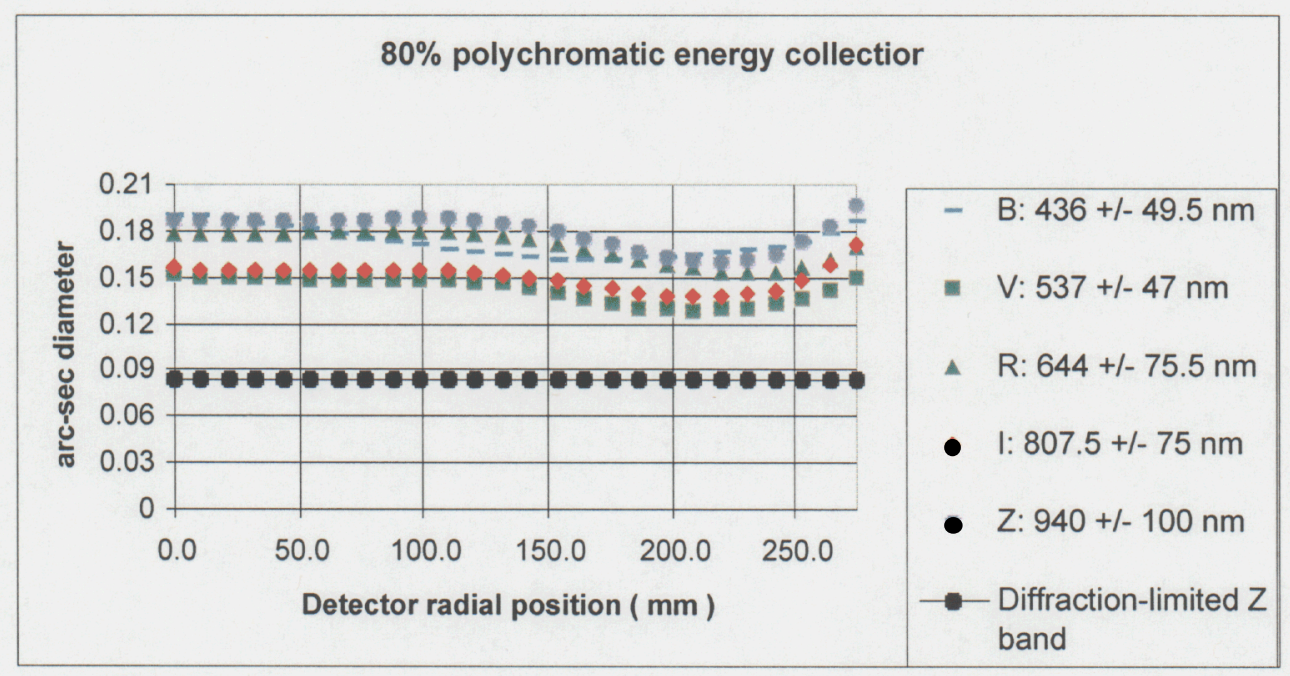

Figure 1 . The image sizes that include $80 \%$ of the polychromatic diffracted energy are plotted vs. radial position on the detector for each spectral band. For comparison, the diffraction-limited $80 \%$ image for the Z-band is about 0.083 arc-sec.

The improvements were made in four main areas. First, earlier LSST designs collected $80 \%$ of the energy in one-third of an arc-second images. This new design, with less than 0.21 arc-second images, represents a $40 \%$ improvement in spot size. Minimal degradation of 0.5 arc-second images will occur. Second, the most difficult optical element to fabricate and test will be the secondary mirror. The Hubble primary mirror and the LSST secondary are about the same size, 3.4 meters in diameter. However, the LSST secondary is convex. This will be the largest precision convex mirror ever manufactured by nearly a factor of two. Current testing procedures will be difficult to adapt for such a large convex mirror. To ease the burden, the aspheric departure of the secondary has been reduced to about 10 microns peak-to-valley from the best-fit sphere. Previous designs had aspheric departures 10x or higher in value. Sommargren and Burge ${ }^{7}$ address testing procedures that are aided by smaller aspheric departures. The other aspheres in this design have reduced complexity and/or reduced departures from the best-fit sphere. The primary mirror is very close to parabolic, and only departs by less than one-half of a micron from the best-fit conic. The tertiary departs only by 137 microns from the bestfit sphere. Both can be tested by an Offner-type null setup. The two largest lenses have aspheric surfaces on the concave surface, with peak-to-valley asphericities of less than 25 microns from the best-fit sphere. The smallest lens is a planoconvex spherical lens. Third, the detector surface is a flat plane, rather than a weakly curved aspheric surface required in previous designs. One can build the detector from a mosaic from many flat CCD detectors that closely match this curved surface. A flat focal plane avoids concerns about how small the detector elements must be to get a good match of the curved surface with acceptable focal errors. A flat detector can have arbitrarily sized CCD elements. The spectral filter is placed as close as possible to this last lens to minimize its size. Finally, the telescope is fully baffled so that no stray light from any field angle, involving a first reflection from the tertiary mirror, can reach the detector.

\section{OPTICAL DESCRIPTION OF THE NEW TELESCOPE}

The optical prescription of the telescope is given in Table 4. The first part of the table gives the design for the R band. The element radii of curvatures, vertex spacing to the next element, inner and outer diameter, and materials are given. The baffles are included as optical elements. The last column gives the aspheric departures from the best-fit sphere, fitted between the inner and outer diameters. The second part of the table gives the aspheric description of each surface, using a standard description of a conic surface plus an additional power series of even terms. The last part of the table gives the changes required to switch to a different spectral band. The amounts of displacement of the primary and tertiary mirrors along with the required spectral filter thickness are tabulated. There are several ways to compensate for focal shifts that also lead to improved focal spot size. In this particular design, three adjustments are required. First, a flat spectral filter of the appropriate band pass is inserted. The filter thickness varies between $21.5 \mathrm{~mm}$ at the blue end to $12.5 \mathrm{~mm}$ in the infrared band. Finally, both the primary and tertiary are repositioned slightly, in opposite directions. The primary moves with a range of one-third of a millimeter. The tertiary moves within a range of about $3 \mathrm{~mm}$. Other possible combinations 
were explored, such as moving the entire detector package and moving one corrector lens. Using only two adjustments, such as moving only the detector package and inserting a different thickness filter, resulted in about $15-20 \%$ larger focal spot sizes at the extreme spectral bands. Many other combinations of three adjustments, such as moving the detector package (detector/corrector lenses/filter) and displacing one lens element along with adjusting filter thickness, yielded similar spot sizes.

Distortion at full field is pincushion with $+0.286 \mathrm{~mm}$ from the paraxial image location, and is mostly third order. This approximately $0.1 \%$ distortion is difficult to correct without seriously affecting the image quality. Correcting the distortion to sub-pixel throughout the field may be nearly impossible. Mapping the distortion field by using known coordinates of stars will be required in any event to determine each pixel location.

The exit pupil is virtual and is located about $5.0 \mathrm{~m}$ past the spectral filter. The optical design of the interference bandpass filter coating could be made simpler by keeping the chief ray angle of incidence zero at all field points; that is, by curving the filters around the exit pupil. Each filter is then a meniscus element with radii of curvature of $5.0 \mathrm{~m}$ concave and convex. The included angle of incidence for an $\mathrm{f} / 1.25$ beam $(\mathrm{NA}=0.4)$ is $+/-23.6$ degrees. For a plane parallel spectral filter, the chief ray has a maximum angle of incidence of about 3.1 degrees. Therefore, curving the filter allows the angle of incidence over which the filter must be designed to decrease from 26.7 degrees to 23.6 degrees. Either choice gives equivalent image sizes. The final shape of the filter will involve a tradeoff between coating and optical fabrication difficulties.

No correction for atmospheric dispersion or ADC has been incorporated. The extremely fast focal ratio and the expected rapid pointing changes during the course of observations preclude any compensation technique. Reduced image quality will have to be accepted at the lower wavelength bands at angles away from the zenith.

\begin{tabular}{|c|c|c|c|c|c|c|c|}
\hline \multicolumn{8}{|c|}{ Optical design data for $\mathbf{R}$ spectral band [all units $\mathbf{m m}$ ] } \\
\hline $\begin{array}{l}\text { Surface } \\
\text { number }\end{array}$ & $\begin{array}{l}\text { Radius of } \\
\text { curvature }\end{array}$ & Spacing & Semi-diameter & $\begin{array}{l}\text { Hole semi- } \\
\text { diameter }\end{array}$ & Material & Description & $\begin{array}{c}\text { Sag from best-fit } \\
\text { sphere }\end{array}$ \\
\hline Outer baffle & & $\begin{array}{l}710 \text { below } \\
\text { secondary }\end{array}$ & 4330 & 2185 & & & \\
\hline 1 & -17897.0 & -5309.996 & 4200 & 2300 & Reflect & primary & $\sim$ parabolic \\
\hline 2 & -6693.0 & 9200.0 & 1700 & 750 & Reflect & secondary & 10 microns \\
\hline Inner bafffle & & $\begin{array}{l}630 \text { above } \\
\text { primary }\end{array}$ & 2175 & 630 & & & \\
\hline 3 & -9466.0 & -4418.0 & 2600 & 450 & Reflect & tertiary & 137 microns \\
\hline 4 & -2065.0 & -48.6 & 625 & none & silica & 1et nenheric lene & \\
\hline 5 & -2902.0 & -384.5 & 625 & none & & ISt asponeric iens & 25 microns \\
\hline 6 & -6682.0 & -20.0 & 465 & none & silica & & \\
\hline 7 & -1669.0 & -239.0 & 440 & none & & 2nd aspheric lens & 25 microns \\
\hline 8 & plano & -16.0 & 340 & none & silica & diahroin & \\
\hline 9 & plano & -22.0 & 340 & none & & dichroic miler & \\
\hline 10 & -2545.0 & -30.1 & 320 & none & silica & & \\
\hline 11 & plano & -50.0 & 320 & none & & 3rd spherical lens & \\
\hline Image & plano & & 275 & none & & detector & \\
\hline
\end{tabular}


Conic constants and polynomial aspheric data

$\begin{array}{cccccc}\begin{array}{c}\text { Surface } \\ \text { number }\end{array} & \text { Conic constant } & \text { 4th order } & \text { 6th order } & \text { 8th order } & \text { 10th order } \\ 1 & -0.99804 & 0 & -2.266 \mathrm{E}-24 & 0 & 0 \\ 2 & -0.1185 & 0 & -8.578 \mathrm{E}-21 & -3.063 \mathrm{E}-28 & -2.275 \mathrm{E}-35 \\ 3 & 0.08228 & 0 & 8.575 \mathrm{E}-23 & 1.527 \mathrm{E}-30 & 0 \\ 5 & 0.4664 & 0 & 4.493 \mathrm{E}-18 & 0 & 0 \\ 7 & -0.3177 & 0 & -3.350 \mathrm{E}-17 & 0 & 0 \\ & & \text { Focal adjustments for each } & \text { spectral band } & \\ \text { Band } & \lambda[\mathrm{nm}] & \text { Primary } & \text { Tertiary } & \text { Filter } \\ \text { B } & 436+1-49.5 & -0.180 & 1.950 & 2.150 \\ \text { V } & 537+1-47.0 & -0.100 & 0.872 & 1.840 \\ \text { R } & 644+1-75.5 & 0.000 & 0.000 & 1.600 \\ \text { I } & 807.5+/-75 & 0.140 & -0.980 & 1.340 \\ Z & 940+/-100 & 0.160 & -1.280 & 1.250\end{array}$

Table 4. Optical design prescription for the $\mathrm{R}$ band, including aspheric terms and baffle location and sizes. The adjustments required to shift spectral bands are also included.

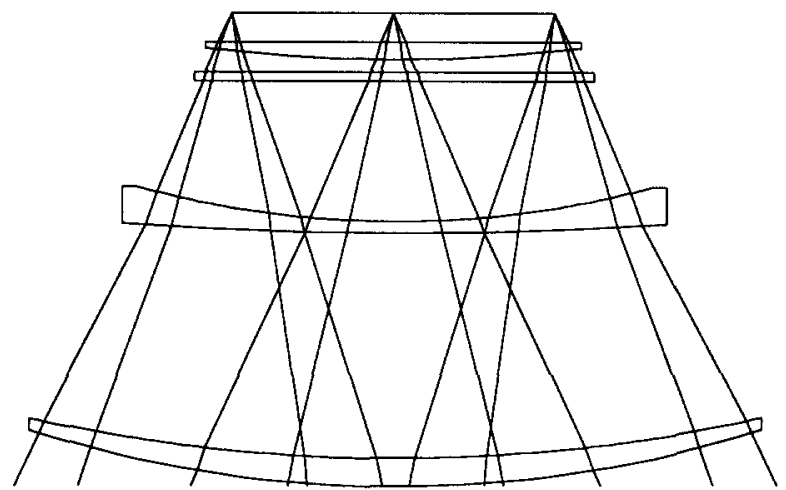

Figure 2 Flat detector, filter and triplet corrector

Optical layout drawings are given in Figure 2 and 3. Figure 2 shows the limiting rays for three points on-axis and at the full field angle. The corrector lenses range in size from $1.3 \mathrm{~m}$ in diameter to $0.64 \mathrm{~m}$. The largest two lenses have aspheric surfaces on the concave surface, with peak-to-valley asphericities of less than 25 microns from the best-fit sphere. The smallest lens is a plano-convex spherical lens. The spectral filter was placed as close as possible to this last lens to minimize its size, about $0.68 \mathrm{~m}$ in diameter. A mechanical filter can be inserted between the first two lenses. There is room to fit the mechanism in without further obscuring the telescope. The optical performance can be improved by either decreasing the axial thickness of components or by lengthening the lens package. However, both changes lead to more difficulties in fabrication since they act to increase the aspect ratio, the diameter-to-thickness ratio.

\section{BAFFLING OF THE TELESCOPE}

Baffling of the telescope is required so that no light that directly reaches the tertiary mirror can be directed onto the detector. Light from angles exceeding the field of view ( $+/-1.5$ degrees) must be considered. Figure 3 shows the limiting rays for an object point on the optical axis. Straight lines just below the secondary mirror (first outer baffle with inner diameter of $4.37 \mathrm{~m}$ ) and just above the primary mirrors (second inner baffle with outer diameter of $4.35 \mathrm{~m}$ ) indicate the clear areas of baffles. The inner second baffle is just slightly smaller in diameter than the hole in the first outer baffle, ensuring that no light from an on-axis point can reach the tertiary. Note that some light can directly reach the tertiary, if the baffles are not in place. Incoming light that misses the primary will be reflected directly from the tertiary 
mirror toward the detector. However, this light is focused near the first corrector lens, sufficiently far from the detector so that the detector hides in the hole of the beam. The detector is protected by the hole in the beam from the defocused beam. For field angles greater than 0.3 degrees, light starts to reach the tertiary with both baffles in place. However, Figure 4 shows the stray light in relation to the $55 \mathrm{~cm}$ detector, ignoring any vignetting by the lens corrector. The small amount of light in the partial annulus comfortably misses the detector, even if the filter and the last lens did not vignette it. The two baffles ensure that, for any field angle, no directly reflected rays can reach the detector.

Previous designs were baffled only so that light from within the specified full field angle could not reach the detector. These designs have collimated beams reflected from the secondary. The angular magnification is about 2.5. Therefore, incoming light at 1.5 degrees $\times 2.5$ magnification, or about 3.75 degrees, looks very much like light reflected from the secondary at full field angle. Therefore, it is necessary to consider light all the way out to about 3.75 degrees when designing baffles. Proper baffling using this criterion would have further increased the off-axis obscuration

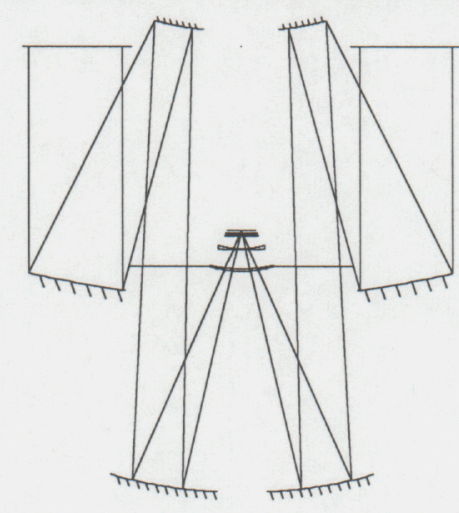

Figure 3. Optical layout of LSST: rays from an on-axis point

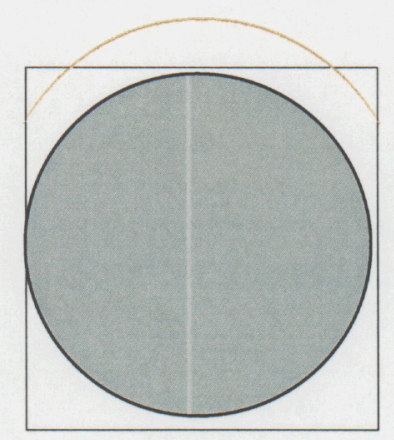

Figure 4. Stray light from 0.3 degrees in relation to the $55 \mathrm{~cm}$ detector

\section{GHOST REFLECTIONS}

Ghosts are not a significant problem. There are a total of 28 double-reflection ghosts from the 8 surfaces of the triplet lens and the filter. Many of these ghosts completely miss the detector because they are so out of focus. An analysis of all double-reflection ghosts showed that the ghost that comes closest to focusing at the detector surface involves two reflections from the spectral filter. Figure 5 shows this double-reflection from each surface of the thinnest spectral filter, $12.5 \mathrm{~mm}$ in thickness. The ghost is a halo $14 \mathrm{~mm}$ in diameter, centered on the assumed 20 -micron image ( 0.4 arc-second image). One can estimate the relative intensity of ghost image to primary image, by comparing relative areas and taking in to account the reflection losses. Simple geometry gives a relationship between the ghost halo of diameter $\mathrm{G}$ and an image of diameter S.

$$
\begin{array}{ll}
I_{\text {rel }}=[S /(1-\varepsilon) G]^{2} R_{1} R_{2}, & S=\text { image diameter }=0.020 \mathrm{~mm} \\
& \varepsilon=\text { linear obscuration ratio }=0.56 \\
& G=\text { ghost image diameter }=14 \mathrm{~mm} \\
& R=\text { surface reflectivities }=0.01 \text { per surface }
\end{array}
$$

$\mathrm{I}_{\text {rel }}=2.0 \times 10-10=\sim 24$ visual magnitude difference

Assuming a $1 \%$ reflection from each surface, the ghost halo is more than 10 orders of magnitude dimmer than the actual image, or a Visual Magnitude difference of 24. Only ghosts from very bright objects will be visible, and even then the ghosts will be spread out over a wide area.

These low reflections can only be achieved if the spectral filter is a combination of an absorbing substrate and a bandpass dielectric coating stack on both surfaces. Filters composed of different band-pass coatings on the front and rear surface may potentially have larger reflection values at particular wavelengths. 


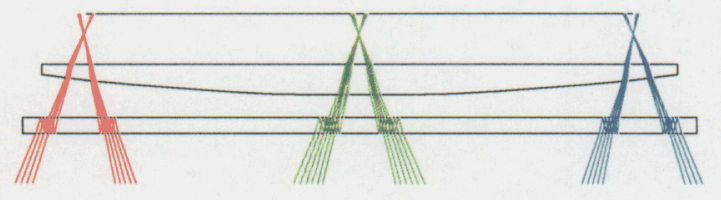

Figure 5. Double-reflection ghost from the spectral filter

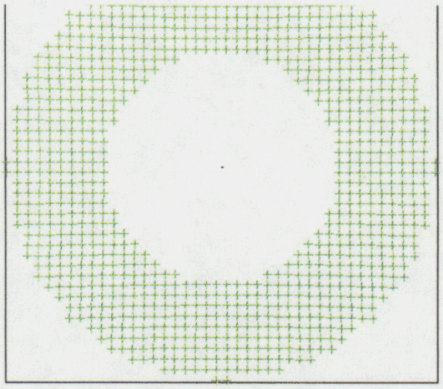

Figure 6. The double-reflection ghost from the filter is $14 \mathrm{~mm}$ in diameter, centered about the 20-micron image

\section{SUMMARY}

In summary, an improved design for the LSST has been presented that shows a $40 \%$ reduction in focal spot size. The secondary mirror and corrector lenses have reduced asphericities that will lead to easier fabrication. The focal plane is flat. The detector can be fully baffled against directly reflected light coming directly from the tertiary mirror. Ghosts will not be a significant problem and can be mitigated by proper design of the spectral filters.

\section{REFERENCES}

1. J. Anthony Tyson and the LSST Collaboration, "Large Synoptic Survey Telescope: Overview", Proc. SPIE 4836 (2002)

2. M. Paul, Rev d 'Optique, 14, p. 169 (1935)

3. R. N. Wilson, Reflecting Telescope Optics I, pp. 215-229, A\& A Library, Springer, 1996

4. J. G. Baker, IEEE Trans. Aerosp. Electron. Syst.,5, 261 (1969)

5. R. V. Willstrop, MNRAS, 210, p597 (1984)

6. R. Angel, M. Lesser \& R. Sarlot, ASP Conference Series, Vol. 195, (2000)

7. G. E. Sommargren and J. H. Burge, "Testing the Large Synoptic Survey Telescope (LSST) Aspheric Convex Secondary", Proc. SPIE 4836-20 (2002) 